\title{
Cobalt Hydride Oxidase Catalysis Enabled Hydroamination of Unactivated Olefins
}

\author{
Wei-Ting Ye ${ }^{1}$ and Rong $\mathrm{Zhu}^{1 *}$ \\ ${ }^{1}$ Beijing National Laboratory for Molecular Sciences, Key Laboratory of Bioorganic Chemistry and \\ Molecular Engineering of Ministry of Education, College of Chemistry and Molecular Engineering, \\ Peking University, Beijing 100871, China
}

\begin{abstract}
Dioxygen is an abundant, selective, and sustainable oxidant that is considered ideal for organic transformations. Oxidative processes using dioxygen as the electron acceptor without oxygen atom incorporation into the substrate are often referred to as oxidase reactions. However, the ground state triplet nature of dioxygen makes such a synthetically valuable pathway incompatible with simple free alkyl radicals, a ubiquitous class of reactive intermediates in the daily synthesis of pharmaceuticals, agrochemicals, and complex natural products. Here we report that a combination of strong cage effect and bimetallic radical-polar crossover successfully addresses this problem, and opens up an oxidase pathway in cobalt hydride catalysis. This leads to a general and chemoselective method that tackles several key challenges in catalytic hydroamination, a fundamental transformation for amine synthesis. Under balloon pressure of dioxygen at ambient temperature, we demonstrate single-step intra- and intermolecular formal addition of a variety of nitrogen nucleophiles, including free amines, sulfonamides, amides, and carbamates, to unactivated alkenes in the presence of a silane, under solventfree conditions. Important medicinal chemistry building blocks such as $\alpha$-branched tertiary amines can be easily accessed, which are often difficult targets otherwise due to their steric hindrance and reducing nature. Mechanistic studies including stoichiometric experiments with well-defined organocobalt complexes provide support for the key hypothesis, which points the way to the development of sustainable processes involving other nucleophiles based on the same design elements.
\end{abstract}


Inspired by the working mechanisms of natural metalloenzymes, catalytic oxidation reactions with dioxygen can be categorize into two classes: oxygenase type, where oxygen atom transfer occurs from dioxygen to the substrate, and oxidase type, where dioxygen serves as an electron/proton acceptor. ${ }^{1,2}$ The latter strategy, in principle, allows any oxidative transformations to be coupled, and is therefore particularly versatile in the pursuit of sustainable processes..$^{3-5}$ However, in practice, the oxidase pathway is found incompatible with a major class of reactive intermediates used in organic synthesis, that is, non-stabilized free alkyl radicals (Figure 1a). This is due to the ground state triplet character of dioxygen, which leads to dominating oxygenation with rate constants approaching the diffusioncontrol limit.6, 7 A prominent example in the context of transition metal catalysis is the well-known Drago-Mukaiyama oxidation reaction. ${ }^{8}, 9$ Hydrogen atom transfer (HAT) from a putative cobalt hydride species to an alkene affords a nascent alkyl radical, which is rapidly intercepted by dioxygen and eventually furnishes ketones or alcohols in high yields.

Nevertheless, we were attracted by the synthetic potential of a "counterintuitive" oxidase pathway, namely an aerobic oxidative carbon-nucleophile bond formation process (Figure 1b). We envisioned that its merge with cobalt hydride-mediated HAT could streamline the sustainable synthesis of a variety of useful chemicals, provided that the innate reactivity toward radical oxygenation could be suppressed. Specifically, we targeted $\alpha$-branched amine derivatives, in particular those bearing a methyl substituent, which play crucial roles in determining the potency and/or selectivity of numerous drug candidates (Figure 1c).10-14 A straightforward strategy to construct these structures is offered by catalytic Markovnikov-selective addition of an amine across an olefin (Figure 1d). Despite of considerable advances in this field over the past twenty years, challenges remain in the reactions of unactivated alkenes, especially in an intermolecular setting. ${ }^{15-21}$ Alternative methods using electrophilic aminating reagents usually necessitate extra steps for either reagent preparation or downstream functional group conversion.22-28 Recently, cobalt hydride-catalyzed oxidative hydroaminations employing stoichiometric I(III) or fluorinating reagents have been reported. ${ }^{29-31}$ While they allow for the direct formal addition of several $\mathrm{N}$-based nucleophiles, the use of specialized strong oxidants has severely 
limited these reactions by high costs, wastes generated, and most importantly, their incompatibility with free amine nucleophiles. In this regard, resorting to an oxidase pathway could lead to an "ideal" hydroamination reaction.

To this end, we hypothesized that the target transformation could be realized by combining two mechanistic features (Figure 1e). First, after HAT, high-efficiency collapse of a metallo/organic radical pair (A) inside a solvent cage might prevent dioxygen trapping. Second, oxidation of the resulting alkylcobalt(III) complex (B) by another molecule of $\mathrm{Co}(\mathrm{III})-\mathrm{X}(\mathrm{X}=$ anionic ligand or counterion) species $(\mathbf{C})$ in the presence of an amine could lead to the amination product, presumably via an innersphere mechanism involving an organocobalt(IV) intermediate. ${ }^{30,32-34}$ The catalyst regeneration would involve aerobic oxidation of $\mathrm{Co}$ (II) species and a series of ligand transfers, which produces silanols as a benign byproduct. The underlying key is to eliminate free alkyl radicals in the reaction system, and isolate the dioxygen reduction from the oxidative functionalization process by invoking two distinct Co(III) species.

Here, we show that cage effect, a long known kinetic phenomenon, can effect a paradigm shift from radical to polar chemistry, which enables efficient intra- and intermolecular oxidative hydroamination reactions at ambient temperature, using dioxygen as a green oxidant and under solvent-free conditions (Figure 1f). Addition of a variety of nitrogen nucleophiles including free amines, sulfonamides, amides, and carbamates to unactivated alkenes are demonstrated. In principle, the scope of this method can be easily extended to encompass other nucleophiles, as showcased by the synthesis of an ether. Notably, acyclic and cyclic $\alpha$-branched tertiary amines can be accessed within a single step from readily available unactivated alkenes and free amines, which are challenging targets otherwise due to their steric hindrance and reducing nature. By this, we show that cobalt hydride oxidase catalysis provides a complementary, step-economical, eco-friendly, and cost-effective approach to a series of pharmaceutically important structures. 
a
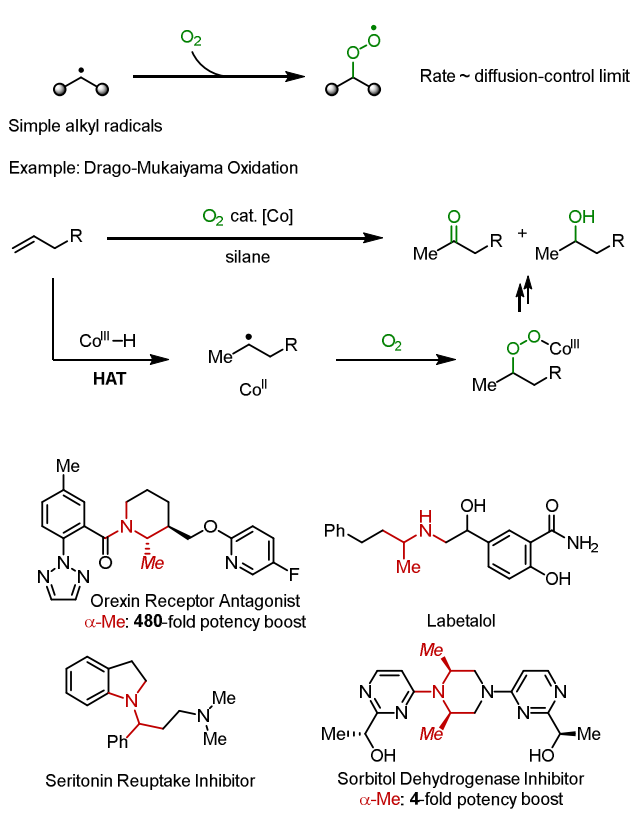

d

- Late TM-Catalyzed Markovnikov Hydroamination via Aminometalation

$ح{ }^{R}+\mathrm{R}_{\mathrm{H}^{-}}^{-R^{2}} \stackrel{[\mathrm{lr}],[\mathrm{Rh}],[\mathrm{Ru}],[\mathrm{Au}],[\mathrm{Pd}], \text { etc. }}{\longrightarrow}$

- MH Catalysis with Electrophilic Aminating Reagents

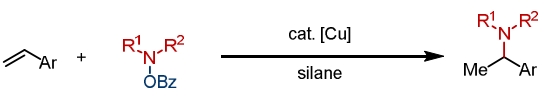

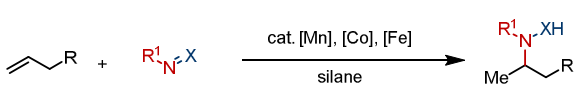

- COH-HAT Oxidative Hydrofunctionalization

$$
\begin{gathered}
\text { Drawbacks } \begin{array}{c}
\text { - Expensive oxidant \& organic wastes } \\
\text { - Incompatible with free amines } \\
\text { (Stoichiometric) }
\end{array} \\
\text { Low efficiency for intermolecular reactions }
\end{gathered}
$$

b
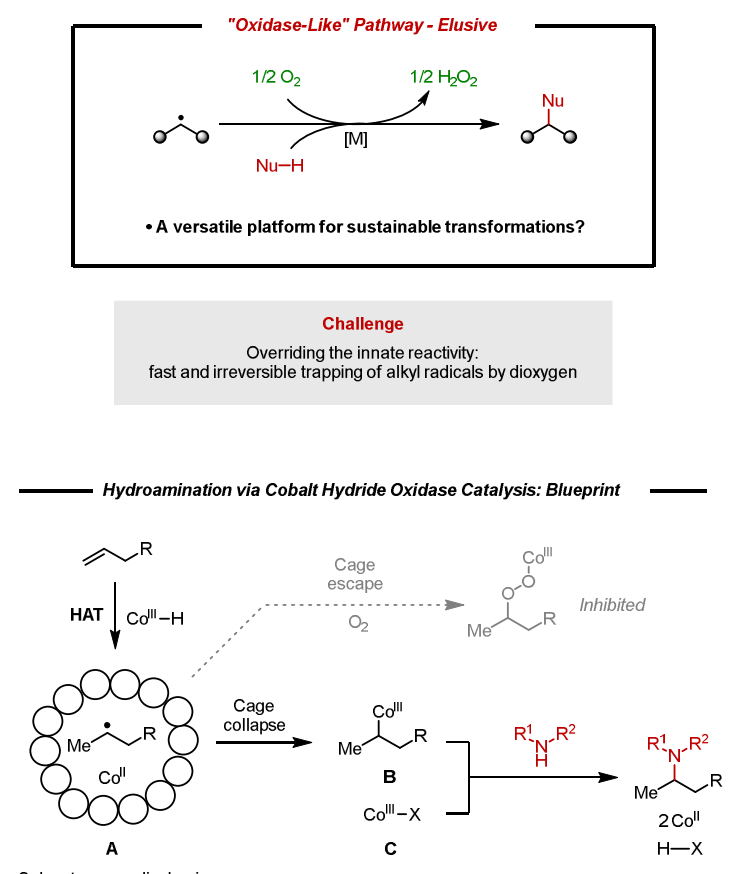

Solvent cage radical pair

KEY 1

- Minimize free radical by maximizing cage effect

KEY 2

- Radical-polar crossover via

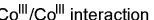

Catalyst turnover:

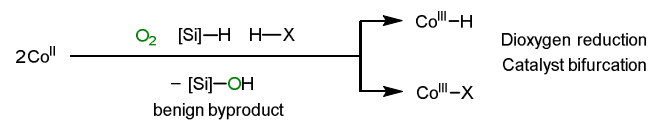

$\mathbf{f}$

This Work: "Oxidase-Type" Intra-and Inter-molecular Oxidative Hydroamination

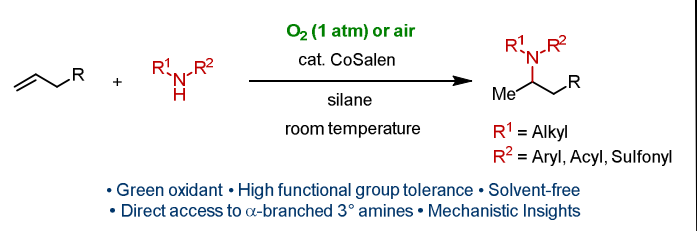

Figure 1. Challenge, significance, and strategy of aerobic hydroamination reactions via cobalt

hydride oxidase catalysis. a. Free alkyl radicals are rapidly trapped by dioxygen, for example, in the Drago-Mukaiyama oxidation reaction. b. The "oxidase-like" pathway to functionalize an alkyl radical is synthetically useful yet challenging. c. $\alpha$-Branched amines/amides are crucial for the potency/selectivity of pharmaceutically relevant molecules. Efficient synthesis of these structures is often elusive due to steric hindrance. d. Late transition-metal-catalyzed Markovnikov-selective hydroamination approaches to $\alpha$-branched amines derivatives based on different pathways and 
aminating reagents. e. Key to use dioxygen as a green and mild oxidant in cobalt hydride-catalyzed oxidative hydroamination: cage effect and radical-polar crossover. f. Intra- and intermolecular hydroamination of unactivated alkenes via cobalt hydride oxidase catalysis (this work).

We commenced our investigation by evaluating the cyclization of a model substrate $\mathbf{2 a}$ in the presence of a catalytic amount of cobalt complex, tetramethyldisiloxane (TMDSO), and dioxygen of balloon pressure $(\sim 1 \mathrm{~atm})$ at ambient temperature (Figure 2a). A few different Co(II) complexes 1a-d were initially tested (entries 1-4). Intramolecular oxidative hydroamination product $\mathbf{3 a}$ were detected along with a number of side products derived from dioxygen trapping (4a, 5a) and hydrogenation (6a) upon HAT/cage escape. We thus defined the selectivity $(S)$ based on eq. (1), which should provide a reasonable estimation of the fractional cage efficiency $\left(F_{c}\right)$, a key characterization of a caged metallo/organic radical pair. ${ }^{35}$ The use of $\mathrm{Co}(\mathrm{III})$ tosylate complexes was then found to give much higher $S$ compared to those obtained with corresponding $\mathrm{Co}(\mathrm{II})$ complexes (entries 5-7). This observation implies that maintaining a high concentration of $\mathrm{Co}(\mathrm{III})-\mathrm{X}$ species could be critical for the oxidase pathway. Along this line, further improvement was achieved by an elevated catalyst loading (entry 8). Interestingly, 1c, a catalyst previously found effective for a number of oxidative hydrofunctionalization reactions, failed to provide $\mathbf{3 a}$ in detectable quantities (entry 3), and an attempt to synthesize the corresponding Co(III) complex was unsuccessful.

Dilution is a common tactic to promote cyclization in the presence of other bimolecular reactions. Notably, cobalt hydride-catalyzed radical cyclizations have been shown to benefit from high dilution by Shenvi and coworkers. ${ }^{36}$ However, the opposite trend was predicted here based on our mechanistic hypothesis for at least two reasons. First, the key oxidation involves two cobalt species and should be facilitated by high concentration, while dioxygen presumably stays at the saturation concentration; second, as the system gets more concentrated, a rise in viscosity would increase the cage efficiency and thereby increase $S$. Indeed, a boost in yield and $S$ was observed as the reactions were carried out using 
less solvent and eventually in neat form (entries 9-10). Such phenomenon also corroborates with our previously observed concentration effect in the reactions promoted by I(III) reagents..$^{30}$

Next, a quaternary ammonium salt was added, which probably increased the solubility of the cobalt complex and potentially facilitated the redox reactions (entry 11). This optimized protocol provided 3a in nearly $90 \%$ yield and the $S$ was over 0.9 , which is close to literature $F_{c}$ values of related organocobalt systems measured in viscous solvents. ${ }^{37}$ We show that when the reaction was carried out under ambient atmosphere, 3 a could be still be obtained in $45 \%$ yield (Figure $2 \mathrm{~b}$ ). Control experiments established the essential roles of the cobalt complex and silane (Figure 2c). It was found that additional base had little effect on the reaction outcome, and toluene sulfonic acid did not effect the cyclization, excluding a proton-catalyzed mechanism. In addition, re-subjecting either $\mathbf{4 a}$ or $\mathbf{5} \mathbf{a}$ to the standard reaction conditions did not produce any $\mathbf{3 a}$, ruling out the in situ conversion of the oxygen-trapping adducts to $3 \mathbf{a}$ (Figure 2d).

a

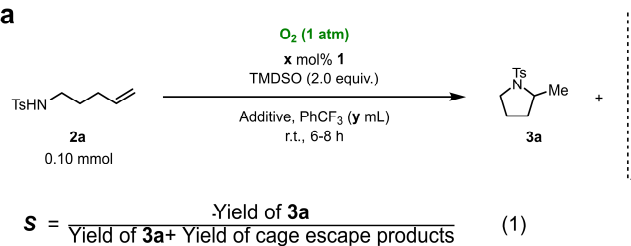

Entry CoSalen $x \quad y \quad$ Additive Conv. (\%) 3 3a (\%) $\begin{aligned} & \text { Cage-Escape } \\ & \text { Products (\%) }\end{aligned}$

$\begin{array}{lllllllll}1 & 1 a & 5 & 1.0 & - & 58 & 22 & 20 & 0.52\end{array}$

$\begin{array}{llllllll}2 & 1 \mathrm{~b} & 5 & 1.0 & - & 14 & 0\end{array}$

$\begin{array}{lllllll}3 & 1 \mathrm{c} & 5 & 1.0 & - & 51 & 12\end{array}$

$\begin{array}{lllllll}4 & 1 \mathrm{~d} & 5 & 1.0 & - & 40 & 16\end{array}$

$\begin{array}{lllllll}5 & 1 \mathrm{a} \cdot \mathrm{O} \text { OTs } & 5 & 1.0 & - & 60 & 29\end{array}$

$\begin{array}{llllllll}6 & 1 \mathrm{c} \cdot \mathrm{OTs} & 5 & 1.0 & - & 27 & 9\end{array}$

$\begin{array}{lllllll}7 & 1 \mathrm{~d} \cdot \mathrm{O} \text { OTs } & 5 & 1.0 & - & 33 & 16\end{array}$

$8 \quad 1 \mathrm{a} \cdot \mathrm{OTs} \quad 10 \quad 1.0 \quad-\quad 100 \quad 61$

$\begin{array}{lllllll}9 & 1 \mathrm{a} \cdot \mathrm{O} \text { OTs } & 10 & 0.10 & - & 100 & 65\end{array}$

$10 \quad 1 \mathrm{a} \cdot \mathrm{OTs} \quad 10 \quad-\quad \ldots \quad 100 \quad 78$

$11 \quad 1 \mathrm{a} \cdot \mathrm{OTs} \quad 10 \quad-\begin{gathered}0.5 \mathrm{eq} . \\ \mathrm{NBu}_{4} \mathrm{OTf}\end{gathered}$

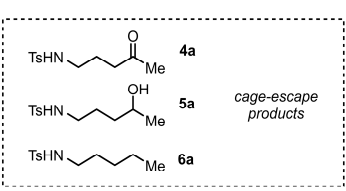

b

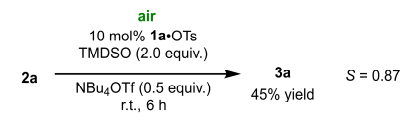

c

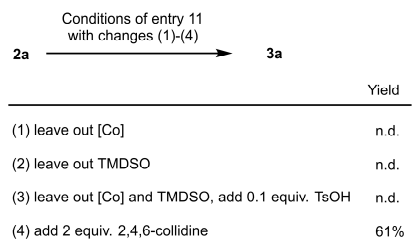

d

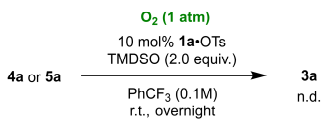

Figure 2. Reaction Development. a Optimization of conditions. Yields and conversions were determined by ${ }^{1} \mathrm{H}$ NMR analysis of the crude reaction mixture. $\mathbf{b}$ The reaction can be carried out under 
ambient atmosphere in moderate yield. $\mathbf{c}$ Control studies. $\mathbf{d}$ The Drago-Mukaiyama oxidation products remained unchanged when resubjected to the reaction conditions.

With the optimized protocol in hand, we set out to evaluate the scope and limitation of this method. Cyclizations with various intramolecular nitrogen-based nucleophiles were examined (Figure 3a). We initially tested several $\delta$-unsaturated sulfonamides and amides, which produced the corresponding $N$ sulfonyl and -acyl pyrrolidines in medium to good yields (3a-d). More useful removable protecting groups such as tert-butyloxycarbonyl (Boc) and benzyloxycarbonyl (Cbz) were also found viable (3eg). Notably, the high functional group compatibility of the method potentially allows for application in complex molecular settings, as demonstrated by the functionalization of a pyrazole-containing drug molecule (3h). In addition to 5-membered rings, this protocol was amenable for synthesizing a challenging aziridine ring, albeit in much lower yield (3i).

As mentioned earlier, a key motivation for developing a dioxygen-based process is to enable the use of free secondary amines, which constitute an important class of nucleophiles that remain to be addressed in hydroamination reactions. ${ }^{38}$ Therefore, we devoted the major part of the study to these substrates, with particular emphasis on $\mathrm{N}$-aryl substituted secondary amines. Despite of the prevalence of dialkylaniline in medicinal chemistry, the introduction of $\alpha$-branching often complicates their synthesis. For example, competing $\beta$-hydride elimination and aryl ether formation become significant in the palladium-catalyzed $N$-arylation of $\alpha$-branched secondary amines. ${ }^{39}$ To this, we demonstrate that 2 methylpyrrolidines bearing a range of electron-rich and -deficient aryl groups at the nitrogen atom could be obtained in high yields, including those containing aryl halides that are reactive toward crosscoupling conditions (3j-p). The effect of additional ortho-methyl substituents on the phenyl moiety was evaluated (3q-r). Such structures are frequently encountered in drug discovery for conformational tuning. The intramolecular hydroamination proved relatively insensitive to steric effect, which renders it a useful complementary approach to these hindered targets. Analogously, piperidine rings were 
prepared, including a hindered 2,6-disubstituted example (3s-u). Although we focused on hydroamination in this work, oxygen-based nucleophiles were briefly surveyed and displayed comparable or even better reactivities. For instance, an unsaturated alcohol cyclized smoothly to afford the corresponding $\alpha$-branched tetrahydrofuran structure under the standard conditions (3v).

Having demonstrated the intramolecular reactions, we turned to the more challenging intermolecular cases (Figure 3b). Directing-group-free methods for such addition using secondary amines and unactivated alkenes are especially lacking. To this end, aerobic cobalt hydride catalysis provides a viable entry. Notably, it does not require a large excess of either reaction partner. Taking the standard protocol without optimization, a series of substituted $N$-methyl anilines were added across simple alkenes smoothly (7a-c). We then evaluated different $N$-alkyl groups on the amine side. $N$-benzylaniline was successfully employed, the product of which can be hydrogenated to reveal a secondary amine (7d). A cyclic amine, namely indoline, displayed similar reactivity (7e), whereas the possible dehydrogenative aromatization was not noticed. Further increasing the steric bulk of the amine led to decreasing reaction efficiency (7f). Nonetheless, useful yield was still obtained using a very bulky nucleophile, $N$ isopropylaniline $(7 \mathrm{~g})$. Besides unactivated alkenes, the scope of this protocol encompasses styrenes, including $\beta$-substituted ones (7j-1). They gave moderate yields as a result of increased oxygenation, which could be attributable to the weaker $\mathrm{Co}-\mathrm{C}$ bond in the corresponding organocobalt(III) intermediates. 
a
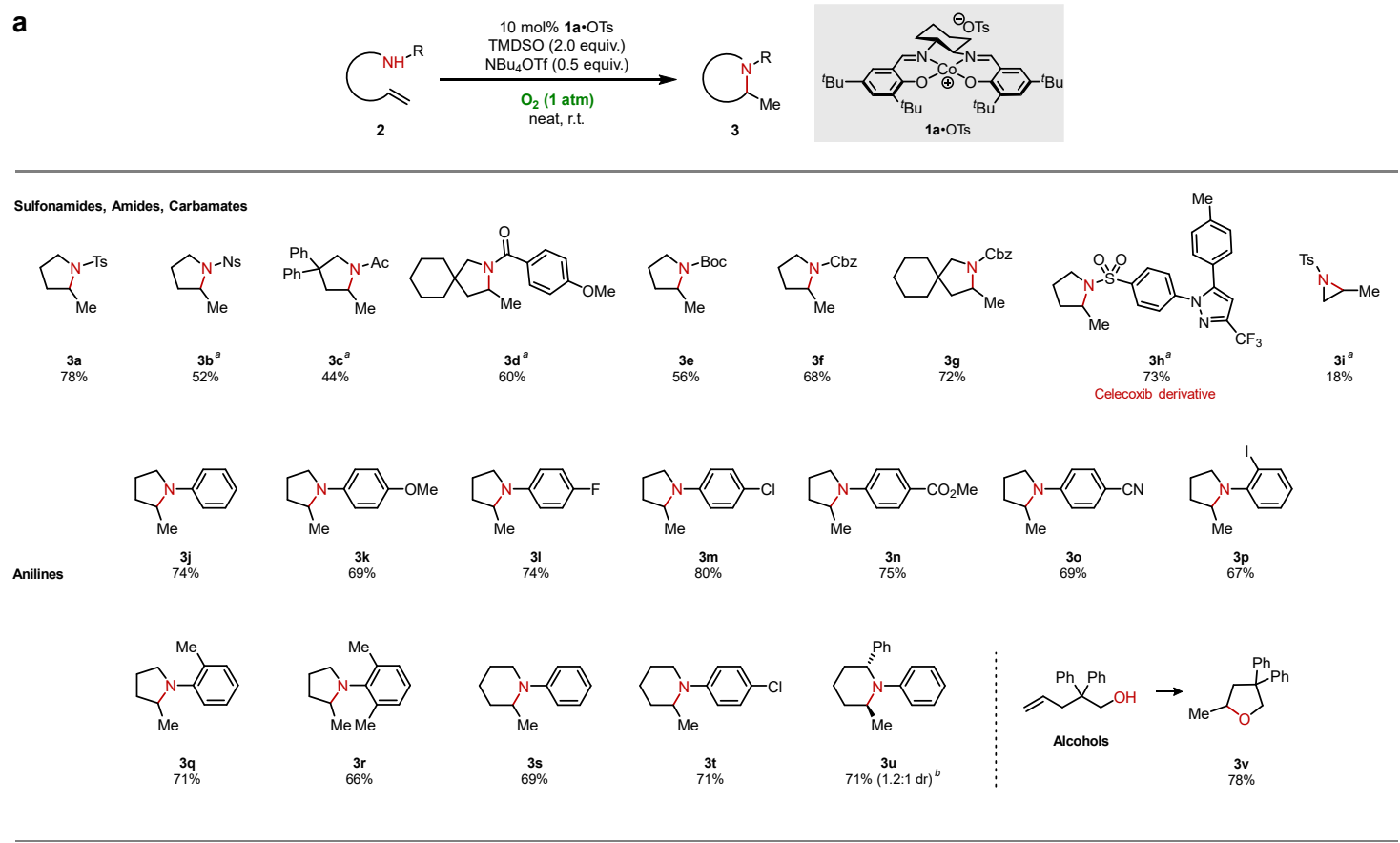

b

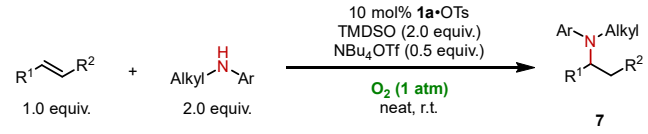
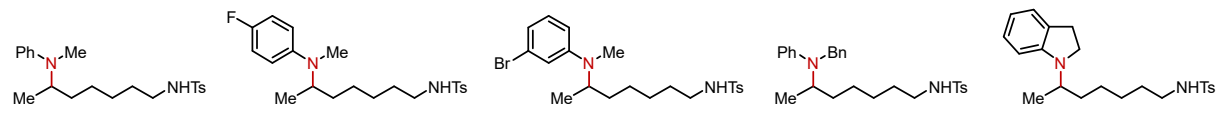

N

$7 a$
$54 \%$

$7 \mathbf{b}$
$53 \%$

$7 c$
$53 \%$

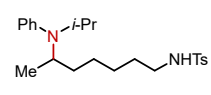

حOPh

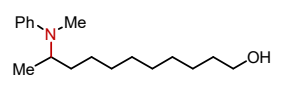

$7 \mathrm{~g}$
$28 \%$

$7 \mathrm{~h}$
$50 \%$

$7 i$
$50 \%$
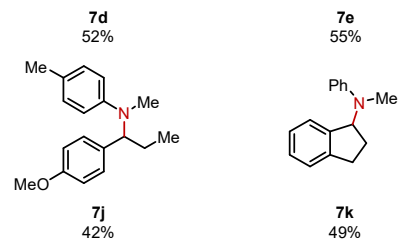

${ }_{41 \%}^{7 f}$

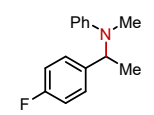

$7 \mathrm{k}$
$49 \%$

$32 \%^{\circ}$

Figure 3. Scope of the aerobic oxidative hydroamination. a Intramolecular addition conditions: 2 (0.30 mmol), $1 \mathrm{a} \bullet \mathrm{OTs}(10 \mathrm{~mol} \%)$, TMDSO (2.0 equiv.), $\mathrm{NBu}_{4} \mathrm{OTf}$ ( 0.50 equiv.), under dioxygen $(\sim 1$ atm) at r.t. for $6-36 \mathrm{~h} . \mathbf{b}$ Intermolecular addition conditions: alkene $(0.30 \mathrm{mmol})$, amine $(0.60 \mathrm{mmol})$ 1a•OTs (10 mol\%), TMDSO (2.0 equiv.), $\mathrm{NBu}_{4} \mathrm{OTf}$ (0.50 equiv.), under dioxygen ( $\sim 1$ atm) at r.t. for 9-14 h. Yields refer to isolated, analytically pure material. a $50 \mu \mathrm{L} \mathrm{PhCF}_{3}$ was added. ${ }^{b} \mathrm{rac}-\mathbf{1} \mathbf{a} \bullet \mathrm{O}$ Ts was 
employed. Diastereomeric ratio was determined by ${ }^{1} \mathrm{H}$ NMR analysis of the crude reaction mixture. ${ }^{c}$ Yield was determined by ${ }^{1} \mathrm{H}$ NMR analysis of the crude reaction mixture.

To gain insight into the reaction mechanism, we systematically evaluated $S$ as a function of concentration, solvent, temperature, and catalyst structure (Figure 4). The reaction of $\mathbf{2 a}$ was selected as a model for study. In $\mathrm{PhCF}_{3}, S$ was found to increase steadily from 0.7 to over 0.9 with an increasing concentration (Figure 4a). Solvent effect was next evaluated (Figure 4b). While no obvious correlation was observed between $S$ and the solvent polarity, a clear trend was noticed that a larger viscosity led to a greater $S$. Notably, isopropanol $(0.1 \mathrm{M})$ provided comparable results with the those obtained under sovlent-free conditions. This supports the hypothesis that the hydroamination proceeds through a cage-collapse pathway, which is favored in viscous medium due to slower diffusion. Elevated temperature promotes the homolysis of the weak $\mathrm{Co}-\mathrm{C}$ bond in an alkylcobalt(III) complex, in addition to lowering the viscosity of the reaction system. This led to an expected decrease in $S$ in both $\mathrm{PhCF}_{3}$ and isopropanol when heating was applied (Figure 4c).

We further prepared dinuclear cobalt(III) salen complexes of similar electronic properties and molecular weights, which contain two different linkages $(\mathbf{1 f} / \mathbf{1 g} \cdot \mathrm{OTs})$. Their catalytic reactivities were studied at different temperatures (Figure 4d). Although their performances were limited by the solubility at ambient temperature, at 40 and $60^{\circ} \mathrm{C}$ they both displayed substantially higher $S$ compared to that for a mononuclear analogue $(1 \mathbf{e} \bullet \mathrm{OTs})$. This observation could be attributable to a few factors. First, according to Noyes' model, radicals of larger size are less prone to escape. ${ }^{40}$ Second, an adjacent cobalt center could offer synergistic effects depending on the oxidation state. A vacant $\mathrm{Co}$ (II) center nearby might increase the chance of cage collapse, whereas a $\mathrm{Co}(\mathrm{III})$ center could presumbaly facilitate the oxidation process. At this stage, the exact origin of this interesting catalyst effect remain unclear. Nonetheless, the length and/or rigidity of the linkage in the dinuclear complex seemed to have an impact. 

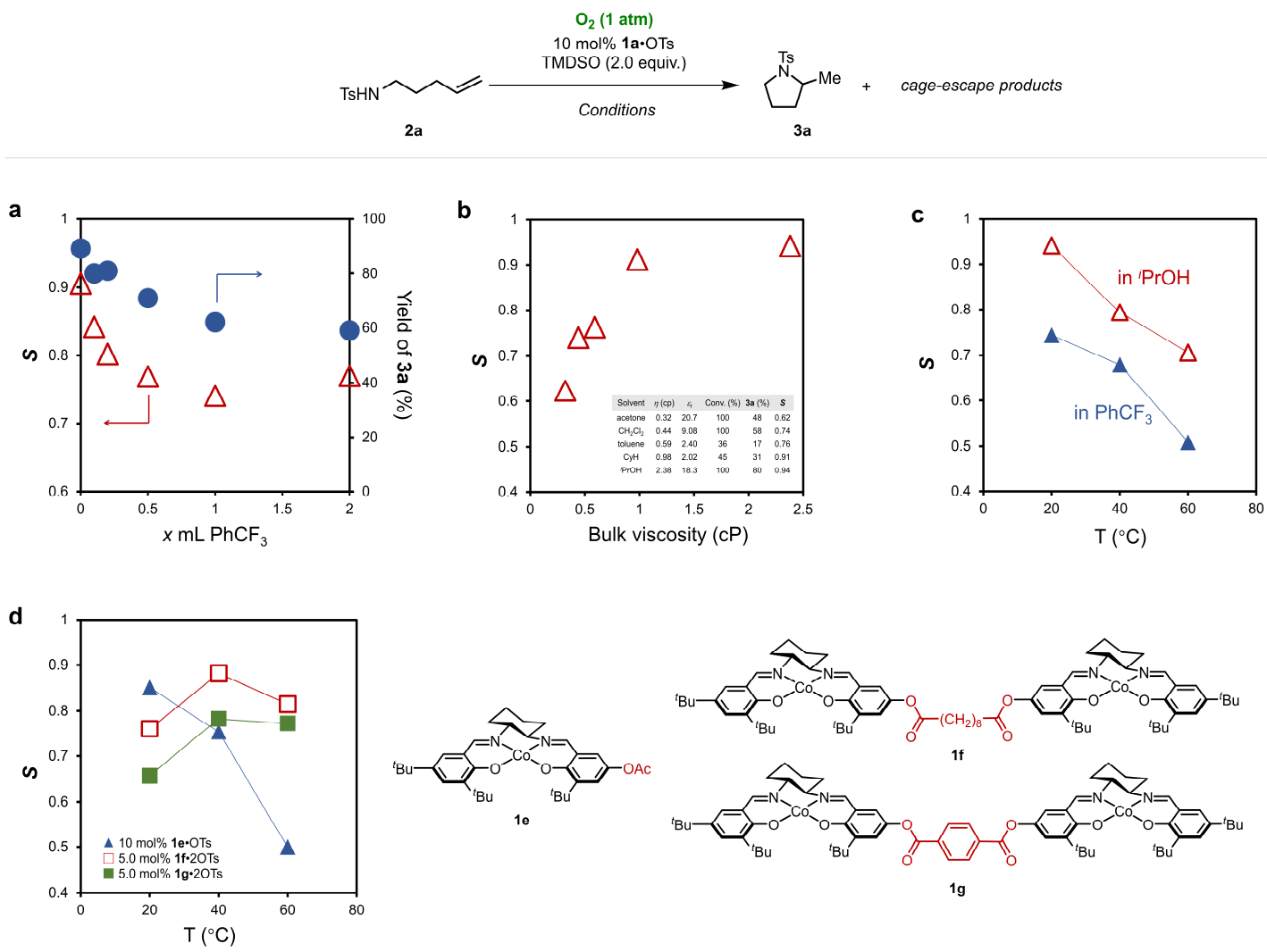

Figure 4. Investigation of $S$ as a function of reaction parameters. All reactions were carried out using $2 \mathbf{a}(0.10 \mathrm{mmol})$, TMDSO (2.0 equiv.), under $\mathrm{O}_{2}(\sim 1 \mathrm{~atm})$ for $6 \mathrm{~h} . S$ were determined by ${ }^{1} \mathrm{H}$ NMR analysis of the crude reaction mixture. a Concentration effect. Conditions: $1 \mathrm{a} \cdot \mathrm{OTs}(10 \mathrm{~mol} \%)$, $\mathrm{NBu}_{4} \mathrm{OTf}(0.50$ equiv.), $(x \mathrm{~mL})$ at r.t. $\mathbf{b}$ Increasing $S$ with increasing solvent viscosity. Conditions: 1a•OTs $(10 \mathrm{~mol} \%)$, solvent $(1.0 \mathrm{~mL})$ at r.t. $\mathbf{c}$ Temperature effect. Conditions: $1 \mathbf{a} \cdot \mathrm{O}$ Ts $(10 \mathrm{~mol} \%)$, $\mathrm{PhCF}_{3}$ or isopropanol $(1.0 \mathrm{~mL})$. d Comparing binuclear and mononuclear catalysts at different temperatures. Conditions: $[\mathrm{Co}](10 \mathrm{~mol} \%), \mathrm{PhCF}_{3}(1.0 \mathrm{~mL})$.

A plausible catalytic cycle is depicted in Figure 5a. To begin with, the HAT process between a cobalt hydride species 9 and an alkene would produce a metallo/secondary alkyl radical pair $\mathbf{1 1}$ inside a solvent cage. Highly efficient cage collapse would generate an alkylcobalt(III) complex $\mathbf{1 2}$, which is critical for inhibiting the classical Drago-Mukaiyama oxidation pathway. Next, electron transfer between $12\left(E_{p}\right.$ 
vs $\left.\mathrm{Fc}^{+} / \mathrm{Fc} \sim-0.01 \mathrm{~V}\right)$ and a cationic $\mathrm{Co}(\mathrm{III})$ complex $10\left(\mathrm{E}_{1 / 2} \mathrm{vs} \mathrm{Fc}^{+} / \mathrm{Fc}=-0.02 \mathrm{~V}\right)$ would lead to a Co(II) complex 8 and a highly electrophilic organocobalt(IV) intermediate 13. ${ }^{34}$ From 13, nucleophilic displacement at the $\alpha$-carbon of the alkyl ligand would finally furnish the hydroamination product, meanwhile producing another molecule of $\mathbf{8}$.

Dioxygen-mediated catalyst regeneration from 8 might begin with an initial formation of a dioxygen bridged binuclear Co(III) species 15 (Figure 5b). Driven by the formation of the strong Si-O bonds, hydride transfer from a silane to $\mathbf{1 5}$ would deliver $\mathbf{9}$ and a disilylperoxide $\mathbf{1 6}$ as suggested by Nojima. ${ }^{41}$ Further reaction of this peroxide with 8 would afford a $\mathrm{Co}(\mathrm{III})$ species $\mathbf{1 7}$, which upon protonation regenerates the cationic $\mathrm{Co}(\mathrm{III})$ species 10 and a silanol 14 as the byproduct. Indeed, when triethylsilane was tested as the hydride source in the model reaction, we noticed that the hydroamination product and triethylsilanol 18 were formed in nearly equal yields.

In order to probe the key oxidative functionalization step, we performed stoichiometric reactions using a well-defined organocobalt(III) complex 19. While no amination was observed when 19 was mixed with excess amount of a secondary amine 20 , with the addition of $1 \mathrm{a} \cdot \mathrm{OT}$, oxidative amination immediately took place at ambient temperature to afford the alkylation product 21 in $28 \%$ yield (Figure 5c). In the meantime, the formation of $\mathrm{Co}(\mathrm{II})$ species was indicated by a color change to brick-red. We next monitored this reaction by UV-Vis spectroscopy (Figure 5d). Initially, the addition of the amine only led to subtle change in the absorption spectrum of 19, presumbly by partial displacing the axial pyridine ligand. This mixture was then titrated by a solution of $1 \mathbf{a}^{\bullet} \mathrm{OT}$, and characteristic absorption bands (360 and $420 \mathrm{~nm}$ ) of Co(II) gradually appeared. Collectively, these data are consistent with our hypothesis that the key carbon-nucleophile bond formation might involve the interaction between two distinct $\mathrm{Co}(\mathrm{III})$ species. 
a

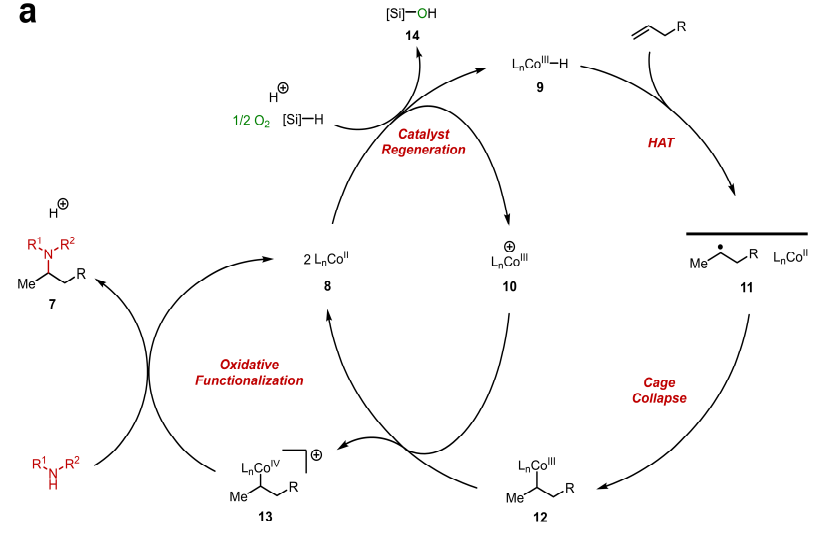

C

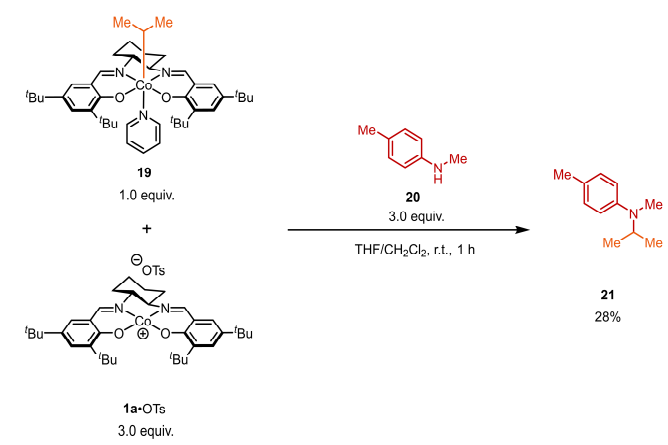

b
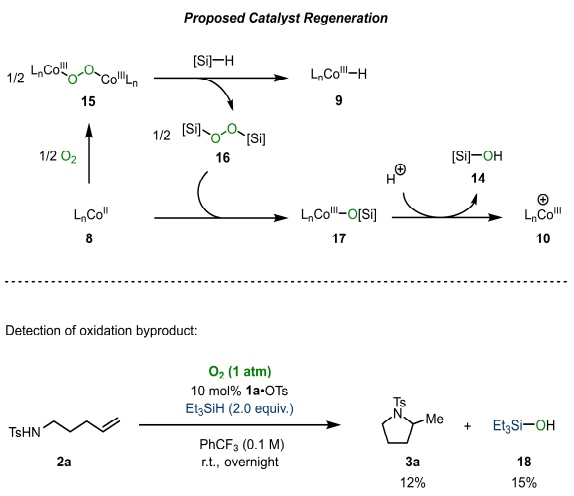

d

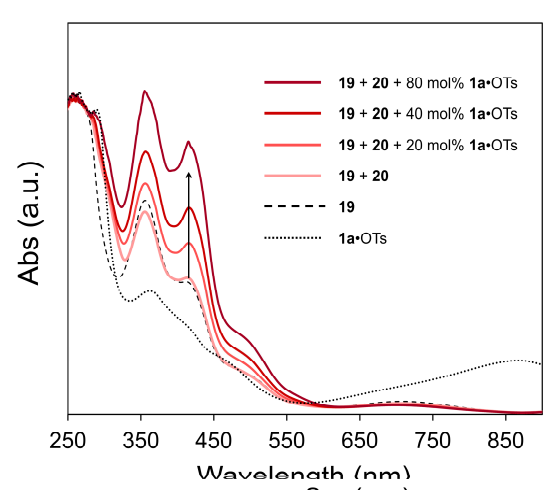

Figure 5. Proposed mechanism and mechanistic experiments. a A possible catalytic cycle involves HAT, cage collapse, oxidative functionalization and aerobic catalyst regeneration. $\mathbf{b}$ A possible mechanism for catalyst regeneration, which is supported by experimental detection of silanols as the oxidation byproduct. c Stoichiometric oxidative amination reaction between a secondary alkylcobalt(III) complex (19) and $1 \mathbf{a}^{\bullet} \mathrm{O}$ Ts in the presence of a secondary amine (20) proceeded smoothly at r.t. to afford a tertiary amine (21). $\mathbf{d}$ Titration experiment in $\mathrm{CH}_{2} \mathrm{Cl}_{2}$ monitored by UV-Vis spectroscopy. To a mixture of 19 (1.0 equiv.) and $\mathbf{2 0}$ (2.0 equiv.) was dropwise added a stock solution of $1 \mathrm{a} \cdot \mathrm{OT}$ Ts at r.t.

In conclusion, we have demonstrated that with a suitable metal catalyst, dioxygen can participate in an oxidase type pathway, where it indirectly oxidizes a simple non-stabilized alkyl radical, rather than trapping it by $\mathrm{C}-\mathrm{O}$ bond formation. Application of this concept in cobalt hydride catalysis leads to 
efficient aerobic intra- and intermolecular oxidative hydroamination, using challenging unactivated alkenes and nitrogen-based nucleophiles including free secondary amines. We anticipate that this method will help to simplify the synthesis of related pharmaceutical building blocks as well as develop more sustainable processes.

\section{References}

1. Stahl, S. S. Palladium oxidase catalysis: selective oxidation of organic chemicals by direct dioxygen-coupled turnover. Angew. Chem. Int. Ed. 43, 3400-3420 (2004).

2. Piera, J. \& Backvall, J. E. Catalytic oxidation of organic substrates by molecular oxygen and hydrogen peroxide by multistep electron transfer--a biomimetic approach. Angew. Chem. Int. Ed. 47, 3506-3523 (2008).

3. Wang, D.-H., Engle, K. M., Shi, B.-F. \& Yu, J.-Q. Ligand-enabled reactivity and selectivity in a synthetically versatile aryl C-H olefination. Science 327, 315-319 (2010).

4. Salazar, C. A. et al. Tailored quinones support high-turnover Pd catalysts for oxidative C$\mathrm{H}$ arylation with $\mathrm{O}_{2}$. Science 370, 1454-1460 (2020).

5. Kim, Y. \& Li, C.-J. Perspectives on green synthesis and catalysis. Green Synth. Catal. 1, $1-11(2020)$.

6. Boukouvalas, J. \& Haynes, R. K. Peroxyl radicals in synthesis. in Radicals in Organic Synthesis: Volume 2 (ed. Renaud, P. \& Sibi, M. P.) 455-484. (Wiley-VCH, 2001).

7. Reed, N. L. \& Yoon, T. P. Oxidase reactions in photoredox catalysis. Chem. Soc. Rev. (2021) doi: 10.1039/D0CS00797H.

8. Zombeck, A., Hamilton, D. E. \& Drago, R. S. Novel catalytic oxidations of terminal olefins by cobalt(II)-Schiff base complexes. J. Am. Chem. Soc. 104, 6782-6784 (1982).

9. Isayama, S. \& Mukaiyama, T. A New method for preparation of alcohols from olefins with molecular oxygen and phenylsilane by the use of bis(acetylacetonato)cobalt(II). Chem. Lett. 18, 1071-1074 (1989).

10. Schonherr, H. \& Cernak, T. Profound methyl effects in drug discovery and a call for new C-H methylation reactions. Angew. Chem. Int. Ed. 52, 12256-12267 (2013).

11. Feng, K. et al. Late-stage oxidative $\mathrm{C}\left(\mathrm{sp}^{3}\right)$-H methylation. Nature 580, 621-627 (2020).

12. Coleman, P. J. et al. Discovery of [(2R,5R)-5-\{[(5-fluoropyridin-2-yl)oxy $]$ methyl $\}-2-$ methylpiperidin-1-yl][5-methyl-2 -(pyrimidin-2-yl)phenyl]methanone (MK-6096): a dual 
orexin receptor antagonist with potent sleep-promoting properties. ChemMedChem 7, 415424, 337 (2012).

13. Chu-Moyer, M. Y. et al. Orally-effective, long-acting sorbitol dehydrogenase inhibitors: synthesis, structure-activity relationships, and in vivo evaluations of novel heterocyclesubstituted piperazino-pyrimidines. J. Med. Chem. 45, 511-528 (2002).

14. Mahaney, P. E. et al. Synthesis and activity of a new class of dual acting norepinephrine and serotonin reuptake inhibitors: 3-(1H-indol-1-yl)-3-arylpropan-1-amines. Bioorg. Med. Chem. 14, 8455-8466 (2006).

15. Xi, Y., Ma, S. \& Hartwig, J. F. Catalytic asymmetric addition of an amine N-H bond across internal alkenes. Nature 588, 254-260 (2020).

16. Ma, S., Hill, C.K., Olen, C. L. \& Hartwig, J. F. Ruthenium-catalyzed hydroamination of unactivated terminal alkenes with stoichiometric amounts of alkene and an ammonia surrogate by sequential oxidation and reduction. J. Am. Chem. Soc. 143, 359-368 (2021).

17. Sevov, C. S., Zhou, J. S. \& Hartwig, J. F. Iridium-catalyzed, intermolecular hydroamination of unactivated alkenes with indoles. J. Am. Chem. Soc. 136, 3200-3207 (2014).

18. Vanable, E. P. et al. Rhodium-catalyzed asymmetric hydroamination of allyl amines. J. Am. Chem. Soc. 141, 739-742 (2019).

19. Bahamonde, A., Al Rifaie, B., Martin-Heras, V., Allen, J. R. \& Sigman, M. S. Enantioselective Markovnikov addition of carbamates to allylic alcohols for the construction of alpha-secondary and alpha-tertiary amines. J. Am. Chem. Soc. 141, 87088711 (2019).

20. Shen, X. \& Buchwald, S. L. Rhodium-catalyzed asymmetric intramolecular hydroamination of unactivated alkenes. Angew. Chem. Int. Ed. 49, 564-567 (2010).

21. Dorta, R., Egli, P., Zürcher, F. \& Togni, A. The [ $\operatorname{IrCl}(\text { Diphosphine) }]_{2} /$ fluoride system. developing catalytic asymmetric olefin hydroamination. J. Am. Chem. Soc. 119, 1085710858 (1997).

22. Zhu, S., Niljianskul, N. \& Buchwald, S. L. Enantio- and regioselective CuH-catalyzed hydroamination of alkenes. J. Am. Chem. Soc. 135, 15746-15749 (2013).

23. Yang, Y., Shi, S. L., Niu, D., Liu, P. \& Buchwald, S. L. Catalytic asymmetric hydroamination of unactivated internal olefins to aliphatic amines. Science 349, 62-66 (2015).

24. Waser, J., Nambu, H. \& Carreira, E. M. Cobalt-catalyzed hydroazidation of olefins: convenient access to alkyl azides. J. Am. Chem. Soc. 127, 8294-8295 (2005). 
25. Gui, J. et al. Practical olefin hydroamination with nitroarenes. Science 348, 886-891 (2015).

26. Xiao, J., He, Y., Ye, F. \& Zhu, S. Remote $\mathrm{sp}^{3} \mathrm{C}-\mathrm{H}$ Amination of Alkenes with Nitroarenes. Chem 4, 1645-1657 (2018).

27. Shen, X. et al. Ligand-promoted cobalt-catalyzed radical hydroamination of alkenes. Nat. Commun. 11, 783 (2020).

28. Jeon, J., Lee, C., Seo, H. \& Hong, S. NiH-Catalyzed Proximal-Selective Hydroamination of Unactivated Alkenes. J. Am. Chem. Soc. 142, 20470-20480 (2020).

29. Shigehisa, H. et al. Catalytic hydroamination of unactivated olefins using a Co catalyst for complex molecule synthesis. J. Am. Chem. Soc. 136, 13534-13537 (2014).

30. Zhou, X. L. et al. Cobalt-Catalyzed Intermolecular Hydrofunctionalization of Alkenes: Evidence for a Bimetallic Pathway. J. Am. Chem. Soc. 141, 7250-7255 (2019).

31. Yahata, K., Kaneko, Y. \& Akai, S. Cobalt-catalyzed intermolecular markovnikov hydroamination of nonactivated olefins: N2-selective alkylation of benzotriazole. Org. Lett. 22, 598-603 (2020).

32. Ebisawa, K. et al. Catalyst- and silane-controlled enantioselective hydrofunctionalization of alkenes by cobalt-catalyzed hydrogen atom transfer and radical-polar crossover. $J$. Am. Chem. Soc. 142, 13481-13490 (2020).

33. Discolo, C. A., Touney, E. E. \& Pronin, S. V. Catalytic Asymmetric radical-polar crossover hydroalkoxylation. J. Am. Chem. Soc. 141, 17527-17532 (2019).

34. Sun, H.-L., Yang, F., Ye, W.-T., Wang, J.-J. \& Zhu, R. Dual cobalt and photoredox catalysis enabled intermolecular oxidative hydrofunctionalization. ACS Catal. 10, 49834989 (2020).

35. Koenig, T. W., Hay, B. P. \& Finke, R. G. Cage effects in organotransition metal chemistry: Their importance in the kinetic estimation of bond dissociation energies in solution. Polyhedron 7, 1499-1516 (1988).

36. Matos, J. L. M. et al. Cycloisomerization of Olefins in Water. Angew. Chem. Int. Ed. 59, 12998-13003 (2020).

37. Garr, C. D. \& Finke, R. G. Radical cage effects in adocobinamide (axial-base-off coenzyme $\mathrm{B} 12)$ : a simple method for trapping $\left[\mathrm{Ado}^{\mathrm{C}} \mathrm{Co}^{\mathrm{II}}\right.$ ] radical pairs. A new $\beta$-H elimination product from the radical pair and measurement of an unprecedentedly large cagerecombination efficiency factor, $F_{c} \geq 0.94$. J. Am. Chem. Soc. 114, 10440-10445 (1992).

38. Musacchio, A. J. et al. Catalytic intermolecular hydroaminations of unactivated olefins with secondary alkyl amines. Science 355, 727-730 (2017). 
39. Park, N. H., Vinogradova, E. V., Surry, D. S. \& Buchwald, S. L. Design of new ligands for the palladium-catalyzed arylation of alpha-branched secondary amines. Angew. Chem. Int. Ed. 54, 8259-8262 (2015).

40. Male, J. L., Lindfors, B. E., Covert, K. J. \& Tyler, D. R. The effect of radical size and mass on the cage recombination efficiency of photochemically generated radical cage pairs. $J$. Am. Chem. Soc. 120, 13176-13186 (1998).

41. Tokuyasu, T., Kunikawa, S., Masuyama, A. \& Nojima, M. Co(III)-alkyl complex- and $\mathrm{Co}(\mathrm{III})$-alkylperoxo complex-catalyzed triethylsilylperoxidation of alkenes with molecular oxygen and triethylsilane. Org. Lett. 4, 3595-3598 (2002).

\section{ACKNOWLEDGEMENTS}

Financial support was provided by the Natural Science Foundation of China (21901011), the Clinical Medicine Plus X Young Scholars Project of Peking University (7100602764) and startup fund from the College of Chemistry and Molecular Engineering, Peking University and BNLMS. The authors would like to thank Yun-Nian Yin and Han-Li Sun (PKU) for help with experiments.

\section{AUTHOR CONTRIBUTIONS}

W.-T. Y and R.Z. conceived the idea, designed the study, performed the experiments, analyzed the data, discussed the result, and wrote the manuscript.

\section{COMPETING INTERESTS}

The authors declare no competing interests. 